\title{
Prophylaxis and therapy of central nervous system involvement in adult acute lymphoblastic leukemia: recommendations of the Polish Adult Leukemia
} Group

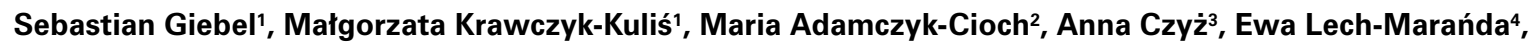 \\ Beata Piątkowska-Jakubas ${ }^{5}$, Monika Paluszewska6, Grażyna Pałynyczko7, Jarosław Piszcz ${ }^{8}$, Jerzy Hołowiecki ${ }^{1}$ \\ ${ }^{1}$ Department of Hematology and Bone Marrow Transplant, Medical University of Silesian, Katowice, Poland \\ 2 Department of Hematology and Bone Marrow Transplant, Medical University, Lublin, Poland \\ ${ }^{3}$ Department of Hematology and Proliferative Diseases of the Hematopoietic System, Medical University, Poznań, Poland \\ ${ }^{4}$ Department of Hematology, Medical University, Łódź, Poland \\ ${ }^{5}$ Department of Hematology, Jagiellonian University School of Medicine, Kraków, Poland \\ ${ }^{6}$ Department of Internal Diseases, Oncology and Hematology, Medical University, Warszawa, Poland \\ ${ }^{7}$ Department of Internal Diseases, Institute of Hematology and Blood Transfusion, Warszawa, Poland \\ ${ }^{8}$ Department of Hematology, Medical University, Białystok, Poland
}

\begin{abstract}
The central nervous system (CNS) is one of the most frequent extramedullary locations of adult acute lymphoblastic leukemia (ALL), affecting approximately $5 \%$ of patients at diagnosis. T-lineage ALL, high initial leukocyte counts and mediastinal involvement are the predisposing factors. In case of relapse, if no prophylaxis was administered, the rate of CNS involvement reaches $30-50 \%$. As the prognosis of patients with isolated or mixed CNS relapse is particularly poor, adequate prophylaxis seems critical. The treatment comprises intrathecal cytostatics, cranial and spinal cord irradiation, as well as systemic chemotherapy including agents penetrating to the CNS. This strategy allows a reduction in CNS relapses to less than $5 \%$ of cases. Compliance to the prophylactic protocols should be one of the principles in the treatment of adult ALL.
\end{abstract}

Key words: acute lymphoblastic leukemia, central nervous system

\section{INTRODUCTION}

Central nervous system (CNS) involvement in acute lymphoblastic leukemia (ALL) patients can be primary or associated with a relapse. This disease location is a direct hazard for life and requires immediate treatment. Moreover, in the case of delay, persistent neurological deficits can occur.

All patients, regardless of the presence of primary CNS involvement, should be treated to prevent leukaemia relapse at this location [1]. The development of the prevention strategies has contributed over the years to a significant improvement in the prognosis in ALL patients. On the other hand, the prevention strategies are not devoid of adverse effects. Some complications can be persistent and contribute to a signifi-

Correspondence to:

Sebastian Giebel, MD, PhD, Klinika Hematologii i Transplatacji Szpiku, Śląski Uniwersytet Medyczny, ul. Dąbrowskiego 25, 40-032 Katowice, Poland, phone: +48-32-256-28-58, fax: +48-32-255-49-85, e-mail: sgiebel@poczta.onet.pl

Received: February 13, 2008. Accepted in final form: April 20, 2008

Conflict of interest: none declared.

Pol Arch Med Wewn. 2008; 118 (6): 356-361

Translated by Sebastian Giebel, MD, PhD

Copyright by Medycyna Praktyczna, Kraków 2008 cant worsening of the quality of life. Therefore, correct therapeutic approach must have adequate efficacy with acceptable toxicity.

\section{Diagnosis}

The diagnosis of CNS involvement in the course of ALL is based on determining the presence of leukaemic cells in the cerebrospinal fluid $[2,3]$. It requires lumbar puncture (LP) and the assessment of cerebrospinal fluid sediment. In doubtful cases, immunophenotypic evaluation using flow cytometry can be useful. If there are absolute contraindications for lumbar puncture, it is considered sufficient to find serious neurological symptoms if they are accompanied by systemic ALL $[2,3]$. In particular, cranial nerve palsy has a special diagnostic value. Central nervous system involvement can also have the form of localised infiltration. When neurological symptoms are present without any abnormalities of the cerebrospinal fluid, it is indicated to perform the imaging examination using nuclear magnetic resonance.

In each case of new ALL diagnosis in adults, diagnostic LP should be performed as soon as possible. In the protocol of 
the Polish Adult Leukemia Group (PALG), the first LP is expected in the pre-treatment period. At this time, thrombocytopenia can be a significant problem interfering with the LP procedure. In this situation, intensive substitution with platelet concentrate is necessary. In the opinion of the authors, platelet levels above $40 \times 10^{9} / \mathrm{l}$, with no accompanying coagulation disorders and signs of diathesis, are sufficient to perform a safe LP.

\section{Primary CNS involvement}

\section{Incidence}

The incidence of primary CNS involvement at the time of ALL diagnosis in adults is estimated at $<10 \%$ [4-6]. In the largest study published to date, performed by the Medical Research Council (MRC, United Kingdom) together with the Easter Cooperative Oncology Group (ECOG, United States), among 1508 patients with new ALL diagnosis, CNS involvement was diagnosed in $77(5 \%)$ patients [5]. In the study of the French group (Group for Research in Adult ALL) and the American group (Southwest Oncology Group) involving 572 and 404 patients, respectively, the incidence was $7 \%$ and $5 \%$ [4,6]. Among 380 patients analysed in the PALG programs, primary CNS involvement was diagnosed in $20(5.3 \%) \mathrm{pa}$ tients. However, it cannot be ruled out that these values are underestimated as they only include patients referred for prospective clinical studies. It should be adopted that some patients with CNS involvement do not meet the inclusion criteria for study protocols due to severe clinical condition, or they are admitted to other departments without an established diagnosis of the underlying disease.

In the light of the analysis by Lazarus et al. [5], the factors predisposing to primary CNS involvement in adults diagnosed with ALL include: 1) high leukocytosis; 2) T-cell ALL; 3) concomitant involvement of the mediastinum. Another study showed higher incidence of CNS involvement in mature B-cell ALL [7]. The presence of the above features particularly indicates the need for early LP.

\section{Treatment}

The basis of treatment in primary CNS involvement in adult ALL patients is intrathecal administration of cytostatics in parallel with remission inducing systemic chemotherapy [1]. In the PALG 5-2007 protocol, methotrexate $15 \mathrm{mg}+$ cytosine arabinoside $40 \mathrm{mg}+$ dexamethasone $4 \mathrm{mg}$ are administered intrathecally. This treatment is used with high possible frequency (1-3 times a week) until the lesions completely regress, that is, until negative results of the cerebrospinal fluid cytomorphological examination are obtained in two consecutive tests.

An alternative for the three drug therapy is the use of cytosine arabinoside in depot form (DepoCyte, Mundipharma, United Kingdom) [8,9]. This product has an improved pene- tration in the CNS, as well as good pharmacokinetics, allowing the maintenance of the therapeutic concentration above the mean inhibitory level for lymphoblasts for at least 2 weeks [10]. Its efficacy has been confirmed in clinical trials in ALL patients and non-Hodgkin's lymphomas with CNS involvement $[8,9]$. When using DepoCyte, LPs are performed every 2 weeks. Due to their large size, the drug particles can be visible during microscopic examination. This fact should be taken into account in the cytomorphological evaluation of the cerebrospinal fluid.

In the MRC/ECOG study, in addition to systemic chemotherapy, it was allowed to irradiate the cranial base with the $24 \mathrm{~Gy}$ dose, and the spinal cord with the 12 Gy dose during phase II induction treatment [5]. As part of the PALG protocol, the possibility of radiation therapy at a dose of 24-35 Gy is expected, with this management being rather reserved for patients resistant to intrathecal treatment. In the remaining cases, scheduled systemic chemotherapy is a priority.

\section{Prognosis}

With correct therapy, the chances to obtain complete remission do not differ significantly for patients with primary CNS involvement compared to the other patients with ALL diagnosis [5,6]. In the MRC/ECOG analysis, the CR rate was $90 \%$, regardless of CNS involvements [5]. Similar results were obtained in the studies of the other working groups [6].

The prognosis as regards the chances for cure is a controversial issue. In the study by Lazarus et al. [5], the chance for survival was significantly lower in patients with CNS involvement $(29 \%$ vs. $38 \%, \mathrm{p}=0.03)$; however, there were no significant differences in the leukemia free survival. On the other hand, however, it should be emphasized that in patients with primary CNS involvement, isolated or mixed recurrences in volving the CNS have been reported significantly more often (11.9\% vs. $5.6 \%$; $=0.04)$ [5].

\section{Leukaemia relapse in the CNS}

\section{Incidence}

Acute lymphoblastic leukaemia relapse in the CNS can be isolated or mixed, i.e. with accompanying infiltration of the bone marrow [11]. Moreover, there is a variant in which bone marrow relapse occurs first, then a secondary CNS involvement is seen. On the other hand, the occurrence of isolated CNS involvement is usually followed by bone marrow involvement. It has been shown that in patients with isolated CNS relapse, even if there are no signs of haematological recurrence, leukaemia cells can be detected in the bone marrow at the level of minimal residual disease [12].

The historical data from the period when there was no CNS focused prevention show that in adults, the incidence of relapse at this location can be $30-50 \%$ [13,14]. In children, this percentage is even higher and it reaches $50-70 \%$ [1]. 
The introduction of the appropriate prevention measures allowed the reduction in the CNS recurrence rate to below $5 \%$ in both age groups [1].

\section{Prevention}

Central nervous system relapse prevention is an important element of the therapeutic program in ALL patients. It includes intrathecal administration of cytostatics, irradiation of the cranial base and the spinal cord, and the use of drugs that penetrate the CNS [1]. With the today's protocols, the CNS recurrence rate is relatively low. In practice, due to this fact, prospective randomised trials with this event as an endpoint are practically impossible. The guidelines for $\mathrm{CNS}$ lesions prevention in adults are therefore mainly based on the experience of the paediatric groups and the expert opinions coming from the comparative analysis of results obtained by each study group.

\section{Intrathecal prevention}

Intrathecal administration of cytostatics is performed in parallel with systemic chemotherapy at each stage of treatment, i.e. during induction, consolidation and remission maintenance [1]. Most study groups prefer the triple therapy (methotrexate, cytosine arabinoside, dexamethasone), and the number of LPs varies and is e.g. 12 in the protocol of the Italian group (Northern-Italian Leukemia Group - NILG) and 14 in the German group (German Multicenter ALL Working Group, GMALL). The Polish Adult Leukemia Group protocol provides the triple therapy used once in pretreatment, twice in the first and second phase of induction, four times during consolidation and every three months in the first year and every 6 months in the second year of maintenance therapy (Fig.). The days of intrathecal administration of the drugs are strictly determined to avoid the overlap of cytotoxicity of administered intrathecally and systemically drugs penetrating to the CNS.

Lumbar punctures are associated with the risk of complications, and, in particular, with post-puncture syndrome characterised by headache, nausea and vomiting that may persist for up to two weeks after LP. The prevention of this syndrome consists in immobilisation in a supine position for at least 6 hours after LP, although some neurologists recommend that this period should be extended to $12 \mathrm{~h}$. The supine position also facilitates drug penetration to the cerebral ventricles [15]. To obtain better distribution, the cytostatics should be dissolved in a sufficient amount of fluid, i.e. at least $6 \mathrm{ml}$ [1].

The studies of the pediatric groups have shown a correlation of so-called traumatic LPs, i.e. those associated with blood extravasation to the spinal canal, a higher rate of CNS relapse and a lower chance for leukemia-free survival [16-18]. This effect is sometimes explained by the possibility of lymphoblasts penetration from the peripheral blood to the cerebrospinal fluid, which is particularly important at the early stage of the disease, before CR is achieved. For this reason,

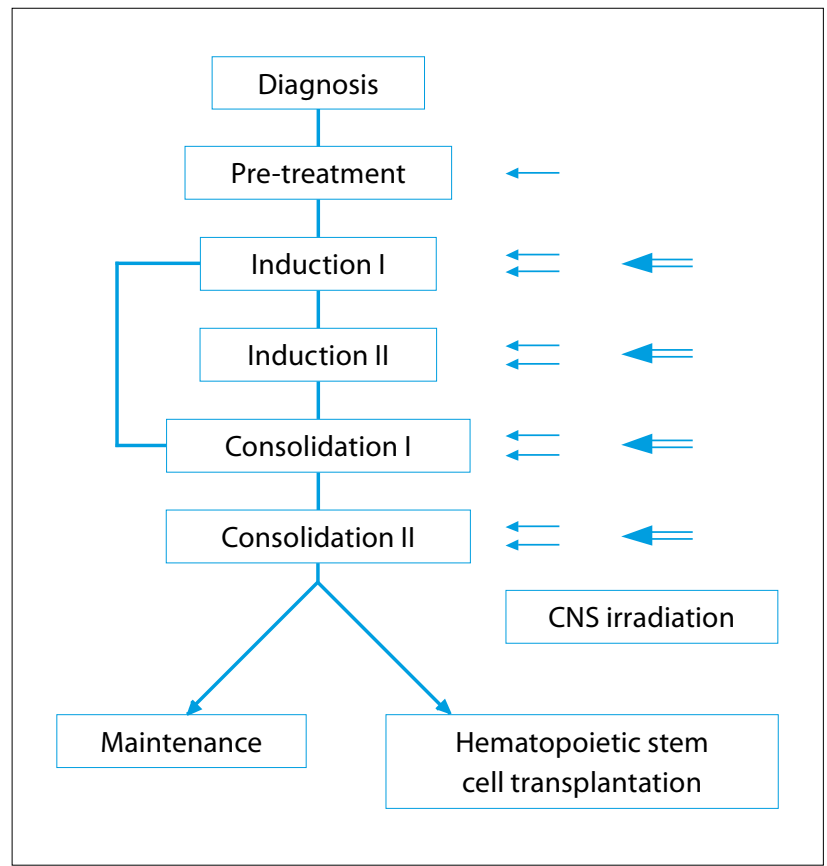

Fig. Prophylaxis of central nervous system relapse in acute lymphoblastic leukemia according to the program of the Polish Adult Leukemia Group (PALG 5-2007). The schedule of intrathecal use of chemotherapy in the pre treatment, induction (depending on the risk factors for relapse, the patients receive one or two courses of induction treatment) and consolidation periods. The arrows indicate lumbar punctures with administration of methotrexate, cytarabine and dexamethasone $(\leftarrow)$ or cytarabine arabinoside in depot form (DepoCyte) $(\Leftarrow)$, as provided in patients with severe post puncture syndrome after the first intrathecal injection

and also due to the risk of other complications, LP should be preceded by a correction of the platelet count and coagulation parameters using the platelet concentrate or freshly frozen plasma, respectively. Coagulation disorders occur very often during induction as a complication associated with the use of L-asparaginase.

Despite the use of the appropriate precautions, some patients experience post puncture syndrome which, if severe, may make the planned intrathecal therapy difficult. In this group of patients, it seems feasible to replace the triple therapy with DepoCyte (Mundipharma, Cambridge, UK), i.e. cytosine arabinoside in depot form. So far, the data on the use of DepoCyte in prevention are isolated single-site reports $[19,20]$. There is no evidence to confirm the efficacy of this treatment from prospective, controlled clinical trials. These studies are currently being conducted in some groups, e.g. the GMALL, the NILG, and the Spanish group (Program for Study and Treatment of Malignant Hemopathies) where the use of DepoCyte is to allow a double reduction in LPs in adult ALL patients, and, as is the case of the GMALL study, to abandon prophylactic irradiation of the CNS. The Polish Adult Leukemia Group protocol provides an opportunity to introduce DepoCyte in place of the triple therapy, with this 
option being reserved for patients who experience symptoms of post-puncture syndrome following the first LP (Fig.). There is a precaution that DepoCyte should not be used concomitantly with high dose cytosine arabinoside (HD-AraC) due to possible cumulative toxicity. The necessary time interval is at least 48 hours after HD-AraC and 14 days before HD-AraC [21]. The possible combination of DepoCyte with the other intrathecal drugs has not been established so far.

\section{CNS irradiation}

Prophylactic CNS irradiation is an effective method to prevent recurrence at this location; however, it is associated with a significant risk of late complications, that is secondary tumors, endocrinopathies, cognitive function disorders and neurotoxicity [22]. As these complications mainly occur in pediatric patients, it is mainly this group where attempts to abandon CNS irradiation or to reduce the dose have been made. In two consecutive studies in which CNS irradiation was abandoned, a relatively high, about $8.3 \%$ and $6 \%$ incidence of isolated or mixed CNS relapse was seen [18,23]. Furthermore, in the study of the Italian group (Gruppo Italiano Malattie Ematologiche Maligne dell'Adulto), in adult patients, CNS irradiation abandonment was associated with $8 \%$ incidence of isolated recurrence [24]. In contrast, another study of the same group in which high dose dexamethasone was used (20 $\mathrm{mg} / \mathrm{m}^{2}$ ), this rate was only $2 \%$ [24]. These data suggest the possibility of abandoning prophylactic CNS irradiation in the case of intensive systemic therapy using drugs that penetrate to the central nervous system. However, this problem remains controversial and most current treatment protocols including the PALG 5-2007 program include CNS irradiation. On the other hand, there is a possibility of abandoning this procedure in the case of patients who are planned to undergo allogenic haematopoietic stem cell transplantation (HSCT) with preparation including total body irradiation. As in the PALG program most patients should be referred for the HSCT procedure, the rate of prophylactic CNS irradiation can be significantly reduced.

\section{Systemic prevention}

A significant component of CNS relapse prevention is the use of sufficiently high doses of drugs penetrating the central nervous system. These include methotrexate, cytosine arabinoside and glucocorticosteroids [1]. The prognostic significance of this form of therapy, as well as the minimum efficient doses of each cytostatic, have not been determined yet. There is the exception of glucocorticosteroid for which it has been shown in pediatric patients that the use of dexamethasone at a dose of $6-6.5 \mathrm{mg} / \mathrm{m}^{2} / 24 \mathrm{~h}$ is associated with a reduced risk of CNS relapse compared to prednisolone at a dose of 40 $\mathrm{mg} / \mathrm{m}^{2}$ [25,26]. Currently, most treatment protocols in adult ALL patients, including the PALG 5-2007 program, provide the use of large doses of methotrexate, cytosine arabinoside

\begin{tabular}{l}
$\begin{array}{l}\text { Table. The most important issues in the prevention } \\
\text { and treatment of central nervous system lesions } \\
\text { in the course of acute lymphoblastic leukemia in adults }\end{array}$ \\
Early diagnosis: lumbar puncture with assessment of cerebrospinal \\
fluid sediment in the pre-treatment period \\
\hline If CNS involvement is diagnosed: intrathecal administration \\
of cytostatics for complete elimination of the blastic cells from \\
the cerebrospinal fluid + irradiation of the cranial base \\
and the spinal cord concomitantly to systemic chemotherapy \\
\hline If no primary CNS involvement is diagnosed: prophylactic use \\
of intrathecal cytostatics in the induction-consolidation period + \\
systemic use of cytostatics that penetrate to the CNS +/- \\
prophylactic irradiation of the cranial base and the spinal cord \\
\hline CNS - central nervous system
\end{tabular}

and dexamethasone, with the intention to both prevent the bone marrow relapse, and CNS recurrence.

The role of high-dose chemotherapy with subsequent hematopoietic stem cell transplant has not been clearly established so far. However, the author's own experience shows that in a significant percentage of ALL patients with prior CNS involvement, the use of autologous bone marrow transplantation prevents recurrence at this location [27].

\section{Treatment}

Central nervous system relapse treatment is similar to the treatment of primary involvement of this system in the course of ALL. As mentioned previously, isolated CNS recurrence almost always precedes systemic relapse [11]. The CNS-focused treatment should therefore be performed in parallel with intravenous chemotherapy, with consideration to cytostatics that penetrate the central nervous system. A particular situation are isolated CNS relapses following allogenic HSCT. In these patients, the graft-versus-leukemia reaction can allow control of the disease in the bone marrow, whereas the CNS is the site of limited availability for immune reactions [28]. The treatment strategy in these patients should take into account close monitoring of bone marrow cell chimerism, and the minimal residual disease status. In justified cases, the treatment can be limited to the CNS, with simultaneous support of the graft-versus-leukemia mechanism by donor lymphocyte infusion, reduced immunosuppression and the use of interferon.

\section{Prognosis}

Although local remission can be obtained in most patients with CNS recurrence, the prognosis in these cases is poor due to concomitant or following bone marrow relapse, often chemotherapy-resistant. In an analysis of 32 patients treated at the M.D. Anderson Cancer Center, diagnosed with isolated or mixed CNS relapse, the 4-year survival prognosis was only 


\section{REVIEW ARTICLES}

$6 \%$ [11]. Furthermore, in the MRC/ECOG study, in a group of 22 patients with isolated CNS recurrence, the 5-year survival was $0 \%$ [5].

\section{SUMMARY}

The prevention and treatment of CNS lesions are among the most important components of the therapeutic strategy in adult ALL patients. The authors' own experience shows that this problem is often underestimated, and the rates of both LPs with intrathecal administration of cytostatics, and CNS irradiation are too low. In light of the presented data, the intensity of prevention seems to be critical, as a CNS relapse is associated with very poor prognosis. Taking this into account, all efforts should be made to complete the entire therapy protocol (Tab.).

\section{REFERENCES}

1. Pui $\mathrm{CH}$. Central nervous system disease in acute lymphoblastic leukemia: prophylaxis and treatment. Hematology Am Soc Hematol Educ Program, 2006; 142-146.

2. Mahmoud HH, Rivera G, Hancock ML, et al. Low leukocyte counts with blast cells in cerebrospinal fluid of children with newly diagnosed acute lymphoblastic leukemia. N Engl J Med. 1993; 329: 314-319.

3. Mastrangelo R, Poplack D, Bleyer A, et al. Report and recommendations of the Rome workshop concerning poor-prognosis acute lymphoblastic leukemia in children: biologic bases for staging, stratification, and treatment. Med Pediatr Oncol. 1986; 14: 194-194.

4. Fière $D$, Lepage $E$, Sebban $C$, et al. Adult acute lymphoblastic leukemia: a multi centric randomized trial testing bone marrow transplantation as postremission therapy. The French Group on Therapy for Adult Acute Lymphoblastic Leukemia. J Clin Oncol. 1993; 11: 1990-2001.

5. Lazarus HM, Richards SM, Chopra R, et al. Central nervous system involvement in adult acute lymphoblastic leukemia at diagnosis: results from the international $\mathrm{ALL}$ trial MRC UKALL-XII/ECOG E2993. Blood. 2006; 108: 465-472.

6. Petersdorf SH, Kopecky KJ, Head DR, et al. Comparison of the L10M consolidation regimen to an alternative regimen including escalating methotrexate/L-asparaginase for adult acute lymphoblastic leukemia: a Southwest Oncology Group Study. Leukemia. 2001; 15: 208-216.

7. Thomas DA, Cortes J, O'Brien S, et al. Hyper-CVAD program in Burkitt's-type adult acute lymphoblastic leukemia. J Clin Oncol. 1999; 17: 2461-2470.

8. Bomgaars L, Geyer JR, Franklin J, et al. Phase I trial of intrathecal liposomal cytarabine in children with neoplastic meningitis. J Clin Oncol. 2004; 22: 3916-3921.

9. Glantz MJ, LaFollette $S$, Jaeckle KA, et al. Randomized trial of a slow-release versus a standard formulation of cytarabine for the intrathecal treatment of lymphomatous meningitis. J Clin Oncol. 1999; 17: 3110-3116.

10. Bleyer WA. Intrathecal depot cytarabine therapy: a welcome addition to a limited armamentarium. Clin Cancer Res. 1999; 5; 3349-3351.

11. Surapaneni UR, Cortes JE, Thomas D. et al. Central nervous system relapse in adults with acute lymphoblastic leukemia. Cancer., 2002; 94: 773-779.

12. Neale GA, Pui $\mathrm{CH}$, Mahmoud $\mathrm{HH}$, et al. Molecular evidence for minimal residual bone marrow disease in children with isolated extra-medullary relapse of T-cell acute lymphoblastic leukemia. Leukemia. 1994; 8: 768-775.

13. Cortes J, O'Brien SM, Pierce $S$, et al. The value of high-dose systemic chemotherapy and intrathecal therapy for central nervous system prophylaxis in different risk groups of adult acute lymphoblastic leukemia. Blood. 1995; 86: 2091-2097.

14. Omura GA, Moffitt S, Vogler WR, Salter MM. Combination chemotherapy of adult acute lymphoblastic leukemia with randomized central nervous system prophylaxis. Blood. 1980; 55: 199-204.

15. Blaney SM, Poplack DG, Godwin K, et al. Effect of body position on ventricular CSF methotrexate concentration following intralumbar administration. J Clin Oncol. 1995; 13: 177-179.

16. Bürger B, Zimmermann M, Mann G, et al. Diagnostic cerebrospinal fluid (CSF) examination in children with acute lymphoblastic leukemia (ALL): significance of low leukocyte counts with blasts or traumatic lumbar puncture. J Clin Oncol. 2003; 21: 184-188.
17. Gajjar A, Harrison PL, Sandlund JT, et al. Traumatic lumbar puncture at diagnosis adversely affects outcome in childhood acute lymphoblastic leukemia. Blood. 2000; 96: 3381-3384.

18. Dutch Childhood Oncology Group, te Loo DM, Kamps WA, Does-van den Berg AV et al. Prognostic significance of blasts in the cerebrospinal fluid without pleiocytosis or a traumatic lumbar puncture in children with acute lymphoblastic leukemia: the experience of the Dutch Childhood Oncology Group. J Clin Oncol. 2006; 24: 2332-2336.

19. Jabbour E, O'Brien S, Kantarjian H. et al. Neurologic complications associated with intrathecal liposomal cytarabine given prophylactically in combination with highdose methotrexate and cytarabine to patients with acute lymphocytic leukemia. Blood. 2007; 109: 3214-3218.

20. McClune B, Buadi FK, Aslam N, Przepiorka D. Intrathecal liposomal cytarabine for prevention of meningeal disease in patients with acute lymphocytic leukemia and high-grade lymphoma. Leuk Lymphoma. 2007; 48: 1849-1851.

21. Pui CH. Toward optimal use of intrathecal liposomal cytarabine. Leuk Lymphoma 2007; 48: 1672-1673.

22. Pui $\mathrm{CH}$, Cheng $\mathrm{C}$, Leung $\mathrm{W}$, et al. Extended follow-up of long-term survivors of childhood acute lymphoblastic leukemia. N Engl J Med. 2003; 349: 640-649.

23. Vilmer $E$, Suciu $S$, Ferster $A$, et al. Long-term results of three randomized trials (58831, 58832, 58881) in childhood acute lymphoblastic leukemia: a CLCG-EORTC report. Leukemia. 2000; 14: 2257-2266.

24. Annino L, Vegna ML, Camera A, et al. Treatment of adult acute lymphoblastic leukemia (ALL): long-term follow-up of the GIMEMA ALL 0288 randomized study. Blood. 2002; 99: 863-871.

25. Bostrom BC, Sensel MR, Sather HN, et al. Dexamethasone versus prednisone and daily oral versus weekly intravenous mercaptopurine for patients with standardrisk acute lymphoblastic leukemia: a report from the Children's Cancer Group. Blood 2003; 101: 3809-3817.

26. Mitchell CD, Richards SM, Kinsey SE, et al. Benefit of dexamethasone compared with prednisolone for childhood acute lymphoblastic leukaemia: results of the UK Medical Research Council ALL 97 randomized trial. Br J Haematol. 2005; 129: 734-745.

27. Hołowiecki J, Krawczyk-Kuliś M, Giebel S, et al. Zajęcie ośrodkowego układu nerwowego w przebiegu ostrej białaczki limfoblastycznej jako czynnik prognostyczny co do nawrotu po autologicznej transplantacji szpiku. Acta Haematol Pol. 1998; 29 (Suppl. 2): 30.

28. Wojnar J, Giebel S, Hołowiecka-Goral A, et al. Impact of chronic graft-versus-host disease on long-term outcome after allogeneic hematopoietic cell transplantation in adult acute lymphoblastic leukemia. Pol Arch Med Wewn. 2006; 116: 671-677. 